\title{
EFFECT OF MOBILITY ON PERFORMANCE OF MACAW MECHANISM OF IEEE 802.11 ADHOC NETWORKS
}

\author{
Ghadeer Hassan Mustafa ${ }^{1}$, Mohamed Essam Khedr ${ }^{2}$ and Ramy Eltarras ${ }^{3}$ \\ ${ }^{1,2}$ Electronics and Communication Engineering Department \\ Arab Academy for Science and Technology Alexandria, Egypt \\ ghadeerhassan2009@gmail.com \\ khedreaast. edu \\ ${ }^{3}$ Computer Science Department \\ Arab Academy for Science and Technology Alexandria, Egypt \\ ramyeast.edu
}

\begin{abstract}
Packet Collisions in wireless communication are one of the most causes of network performance degradation. MACAW mechanism is a popular and widely used mechanism to reduce packet collisions that arise due to the hidden terminal problem in mobile ad-hoc networks. However; Mobility is considered a vital issue in mobile ad-hoc networks that has not been thoroughly investigated. A real time simulation is carried out to measure the Throughput and the Packet Delivery Ratio of an ad-hoc network under Shadowing propagation model using MACAW mechanism with different mobility scenarios.
\end{abstract}

\section{KEYWORDS}

Mobile Ad-hoc network, MACAW mechansim, IEEE 802.11b, Shadowing

\section{INTRODUCTION}

Wireless Communication has impacted our daily lives and has become significant for our modern existence. Drastic changes have taken place since the early development of radio telephony to current smart devices such as tablets, iPhones that support communication with higher data rate, coping with the increasing demand for mobility and flexibility in nowadays lifestyle. In addition to wireless multimedia that is becoming increasingly popular as they offer users the accessibility to information and multimedia services anytime. As wireless communication continues to evolve, the future holds many more possibilities in this field.

IEEE 802.11 is considered to be an important standard for Wireless Local Area Networks (WLANs) which is adopted by many manufacturers of WLAN products. IEEE 802.11 is concerned with the physical layer and the MAC layer. The physical layer is in charge of transmitting the raw data over Radio Frequency (RF). Whereas, the MAC layer coordinates access

Dhinaharan Nagamalai et al. (Eds) : CSE, DBDM, CCNET, AIFL, SCOM, CICS, CSIP- 2014

pp. 251-259, 2014. (C) CS \& IT-CSCP 2014

DOI : $10.5121 /$ csit.2014.4422 
to a shared radio channel and guarantees the privacy of the transmitted data as well as the reliability of the data services. In addition to management protocols that ensure authentication and data delivery. In contrast to other LAN standards, wireless standards address unique issues such as node mobility, limited Bandwidth availability, error prone broadcast channel, power management, link reliability management and hidden and exposed terminal problems, none of which was a concern for other standards in IEEE 802.

The IEEE 802.11 has attracted many researchers to analytically model its access mechanism due to its wide popularity. Network designers were able to ascertain proper values for different parameters that lead to the best performance; also they were able to decide efficiently on the network design taking into consideration the required performance and the expected traffic. Finally, researchers have proven the efficiency of new mechanisms that were proposed to enhance the 802.11 performance. [7]

One of the major challenges that faced researchers was the impact of mobility on wireless ad-hoc networks. Mobile ad-hoc networks lack fixed topology due to node mobility, interference, path loss, shadowing. As a result of this challenging environment, rapid and random changes of the network topology at unpredictable times take place.

The main contribution of this paper is to provide a real time simulation of a randomly distributed mobile ad-hoc networks under Shadowing propagation model in order to compute the Throughput and the Packet Delivery Ratio with different mobility scenarios and different network loads.

This paper is organized as follows. First, Section II gives a review study on related work. Section III describes the system implemented. Section IV demonstrates the system design with simulations and finally conclude the paper in Section V.

\section{RELATED WORK}

In [7], Mohand and Louiza introduced an extension of the Bianchi's Markov chain model with Packet Fragmentation Mechanism (PFM) and the Packet Error Rate (PER). A mathematical model was presented to compute the overall throughput and the mean response time of the IEEE $802.11 \mathrm{~b}$ and the results were validated by simulation. The authors focused on how to improve the performance achieved in IEEE 802.11b Distributed Coordination Function (DCF) network by using the packet fragmentation mechanism under the assumption of non- ideal channel and infinite load conditions. The introduced analytical results have been carried out for different Bit Error Rate (BER) values, packet lengths, and network sizes and data rates.

In [6],Guowang, Ye and Ananthram presented a multistage channel aware aloha scheme for the contention period that allows users with relatively better channel states to have a higher probability of contention success while assuring fairness among users. The authors were able to analytically prove that the proposed scheme resolves network contention and attains throughput close to that of centralized schedulers. The scheme is also robust to any uncertainty in channel estimation. Simulation results were carried out to demonstrate that the proposed scheme significantly improves network performance in comparison to other existing scheme such as 802.11 RTS/CTS scheme. 
In [3],Abderrahim and Abderrezak introduced a scheme called Relative Fairness and Optimized Throughput (RFOT) that ensures fairness and allows each node to adapt its transmission rate and contention window size to channel quality. The propsed scheme was validated using analytical model based on a 3-dimension Markov chain. The authors were able to prove that their scheme achieved fairness without comprising the throughput even under high load network.

Through the reviews presented previously, it is obvious that many researchers were interested to analyse and enhance the performance of IEEE 802.11.

\section{SYSTEM DESCRIPTION}

The performance of wireless network is dependent on medium access control (MAC) protocol used. Carrier sense multiple access (CSMA) is widely used due to its scalability and simplicity. CSMA is prone to hidden terminal problem especially in ad-hoc networks where there is a direct communication between nodes. Hidden nodes result in collision of packets which leads to degradation in network performance. As a solution to such problem, MACAW mechanism was introduced. [8]

In MACAW mechanism; when a node attempts to transmit data, it first sends a short control packet called Request To Send (RTS) to the receiver. The RTS packet includes the source, destination and the duration of the whole data transmission. This packet is received by all users that lie within the transmitter's range. Every node upon receiving the RTS sets it's virtual carrier sense indicator called Network Allocation Vector (NAV). The NAV has a time value that represents the duration up to which the wireless medium is expected to be busy because of an ongoing transmission. Therefore; every packet has the duration information for the remainder of the message. Every node overhearing a packet continuously updates its own NAV. After waiting for Short Inter-frame Spacing (SIFS), the receiver answers with a response control packet called Clear To Send (CTS).[8]

All users receiving either RTS or CTS automatically set their NAV for the given duration and then use it together with the Physical carrier sense to sense the medium. Once the RTS packet has been sent and CTS packet has been received successfully, all nodes within receiving distance from the sender and from the receiver are informed that the medium is reserved for one sender exclusively.[3] After waiting for SIFS, the sender starts data packet transmission. Upon receiving the data the receiver waits an additional SIFS and then sends and an ACK. After this four handshake process the transmission is over and the NAV in each node is marked as free and the process can repeat again.

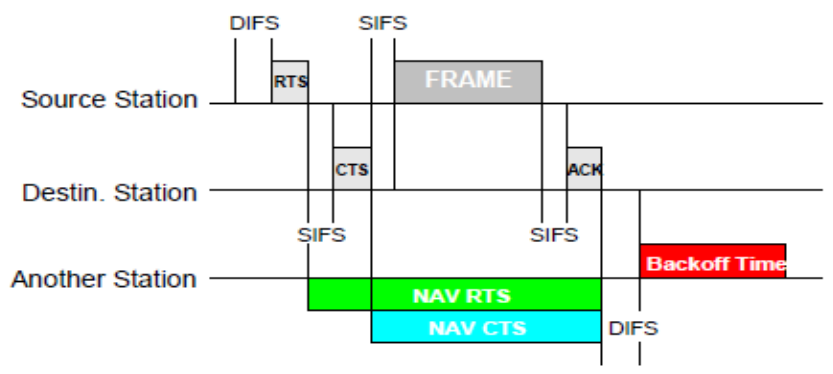

Fig.1. MACAW mechanism 
Though; MACAW mechanism ensured packet delivery through the addition of the RTS/CTS packets, mobility of users was still a major issue that questioned such efficiency. Therefore, Mobility management becomes an interesting area of research as the mobile user should benefit from smooth handoff and low packet drops. It is considered a key participant in efficiently delivering data to mobile users. The main use of mobility management is to track, store and update the location information of the mobile user that move from one place to another within a certain coverage area of single wireless mobile ad-hoc network or within multiple wireless mobile ad-hoc networks in order to allocate the right amount of resources.

Fading is a vital part of a wireless communication design. Fading can be defined as a rapid fluctuation of the received signal strength over short time intervals. As the signal propagates through the wireless medium, it is faced with several obstructions, for example buildings, walls and other objects. These so called physical obstructions make the transmitted signal encounters signal attenuation. Therefore, Shadow fading can be defined as the variation in the received signal power due to these obstructions. Shadow fading is a fading that occurs on a large scale. In general, the variation in the received power due to shadow fading follows a Gaussian distribution.

$$
\text { From Equation } P_{r=} \emptyset_{r} a=\left(\frac{P_{t}}{4 \pi d^{2}}\right) a
$$

Where $P_{t}$ is the radiated power from an isotropic transmitter, ais the effective area of the receiving antenna, $P_{r}$ is the captured power and finally $\mathrm{d}$ is the distance where the receiver is located. Even though $d$ is the same in the previous equation, the received power still varies since some locations face greater shadow fading than do others. In order to resolve this issue, the transmitted power should be increased to compensate for the shadow fading effect.

In our wireless system design, we were able to simulate the MACAW mechanism through designing a network with randomly distributed mobile users under Shadowing propagation model. Each user has a $40 \mathrm{~m}$ range in a $100 \mathrm{~m}$ square area as shown in figure

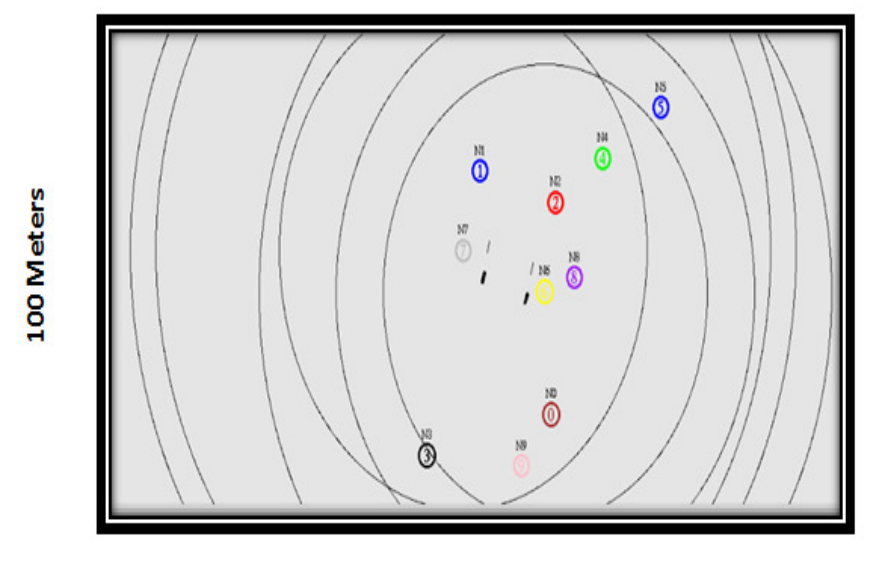

100 Meters

Fig.2. Randomly Distributed Mobile Users 


\section{SiMULATION RESULTS}

In the network research field, deploying a complete test beds that are composed of multiple networked computers, routers and data links to endorse and substantiate a certain network protocol or a specific network algorithm are extremely expensive. Therefore, the use of network simulators in such cases saves a lot of money and time in achieving this task. In this section, we demonstrate the performance of MACAW mechanism using Network Simulator Tool (NS2).[4]

NS2 is considered one of the most popular open source network simulator tool used among researchers.[9] It is an object oriented, discrete event driven network simulator that was developed at UC Berkeley as a part of Virtual Internet Test beds (VINT) project. Ns2 uses two languages $\mathrm{C}++$ and OTCL (TCL script language with Object-oriented extension developed at MIT). [4] Therefore, the presence of these two languages has proven to be more effective. A simplified user's overview of Ns2 is shown below.

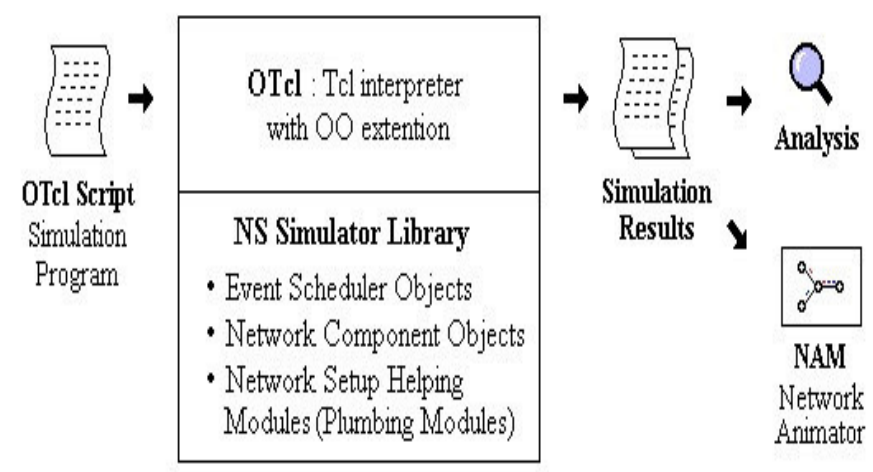

Fig.3. A Simplified User's Overview of Ns2

Simulation results are stored as trace files in Ns2 and a visual overview of the network is displayed using the Network Animator (NAM). A flow chart that highlights the flow of events for a Tcl script file runs in Ns2.

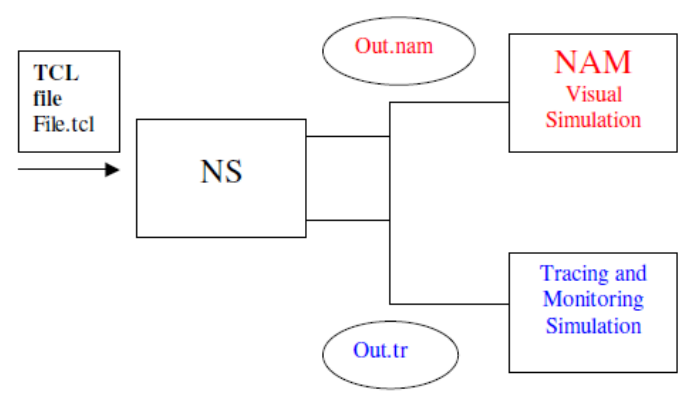

Fig.4. Flow of events for a Tcl file Run in Ns2

The performance of MACAW mechanism is determined by certain parameters such as Throughput and Packet Delivery Ratio (PDR). 
Throughput is the measure of the actual data sent per unit time across a network in the real world.

$$
\text { Throughput }=\left(\frac{\text { Total number of Bytes received }}{\text { Total Time duration of each packet }}\right)
$$

PDR is the ratio of the data packets successfully delivered to the destination to the packets generated by the sender. [2]

$$
P D R=\left(\frac{\text { Received Packets }}{\text { Sent Packets }}\right) * 100 \%
$$

Consider the network topology given in Fig.2.In each simulation trial, mobile users are randomly distributed under Shadowing propagation model in a $100 \mathrm{~m}$ square area. Each user has a $40 \mathrm{~m}$ transmission range and sender-destination pair is selected randomly among users for data transmission. The different parameters that were used to set up our network design are illustrated by both Table.I. and Table.II.

TABLE.I Simulation Setup Parameters

\begin{tabular}{|l|l|}
\hline \multicolumn{1}{|c|}{ Parameters } & Values \\
\hline Area & $100 \times 100 \mathrm{~m}^{2}$ \\
\hline Transmission Range & $40 \mathrm{~m}$ \\
\hline $\begin{array}{l}\text { Number of randomly } \\
\text { distributed users }\end{array}$ & $5,10,15,20$ users \\
\hline Physical/MAC layer & IEEE $802.11 \mathrm{~b}$ at 1Mbps \\
\hline Mobility Model & $\begin{array}{l}\text { Random way point with } \\
\text { pause time }\end{array}$ \\
\hline Pause Time & $2.0 \mathrm{~s}$ \\
\hline Maximum Mobility Speed & $10 \mathrm{~m} / \mathrm{s}$ \\
\hline Packet size & 512 Bytes \\
\hline Traffic Type & $\begin{array}{l}\text { TCP(Transmission Control } \\
\text { Protocol) }\end{array}$ \\
\hline Simulation Time & $200 \mathrm{~s}$ \\
\hline Number of Simulation Runs & 30 \\
\hline
\end{tabular}

Fig.5. investigates the influence of network load on Throughput; we run simulation with 5, 10, 15, and 20 randomly distributed mobile users. For each case, we run 30 trials for 200s; each packet has a size of 512 bytes. We observe that network designed with 5 users had the least throughput of $0.3608 \mathrm{Mb} / \mathrm{s}$. Upon increasing the number of users to 10 , a significant increase in the throughput of $0.4149 \mathrm{Mb} / \mathrm{s}$ was noticed. A further increase in the network load where the numbers of users were increased to 15 users led to a slight decrease in throughput value that reached $0.4148 \mathrm{Mb} / \mathrm{s}$. Finally, the maximum throughput of $0.4194 \mathrm{Mb} / \mathrm{s}$ was achieved at a heavier network load of 20 Users. 


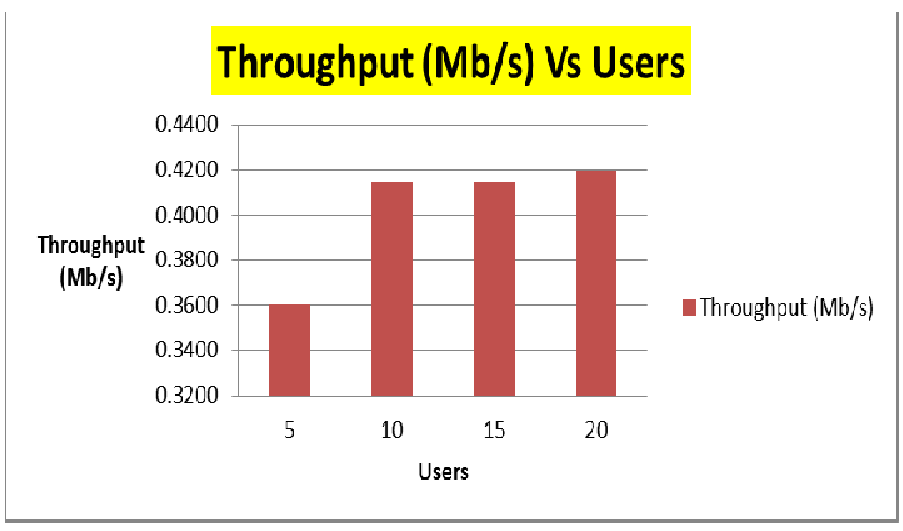

Fig.5.Average Throughput versus Randomly Distributed Mobile Users

Fig.6. shows the impact of network load on the Packet Delivery Ratio. In order to verify this impact, we run simulation with 5, 10, 15, and 20 randomly distributed mobile users. For each case, 30 trials were carried out for 200 s, we see that, network designed with the least number of users had the least PDR of $91.9238 \%$. As we increase the number of users to 10 users, a significant rise in the PDR value that reached $93.0086 \%$. A noticeable drop in the PDR value of $92.7957 \%$ took place when the number of users was increased to 15 users and this reflects the drop in throughput that took place in Fig.5. Finally, the maximum PDR was reached at $93.0298 \%$ when the maximum number of users was used.

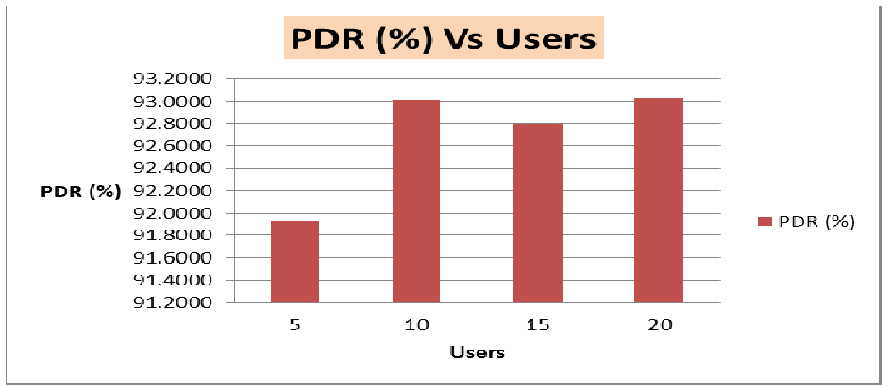

Fig.6.Average PDR versus Randomly Distributed Mobile Users

Fig.7. shows how mobility of nodes has a major impact on the overall network throughput. We run simulation with maximum speed of $5,10,15$, and $20 \mathrm{~m} / \mathrm{s}$. For each case, we compute the average speed of nodes and run our simulation for 30 trials, each with duration time of 200s. Fig.7. shows that at average speed of $2.5 \mathrm{~m} / \mathrm{s}$, a maximum throughput of $0.4257 \mathrm{Mb} / \mathrm{s}$ was achieved. A further increase in the average speed of nodes led to a drop in the overall network throughput that has reached a value of $0.4081 \mathrm{Mb} / \mathrm{s}$ at $5.0 \mathrm{~m} / \mathrm{s}$. A slight increase in the overall network throughput value of $0.4097 \mathrm{Mb} / \mathrm{s}$ was noticed at average speed of $7.5 \mathrm{~m} / \mathrm{s}$. Finally, the minimum throughput of $0.4007 \mathrm{Mb} / \mathrm{s}$ took place at average speed of $10 \mathrm{~m} / \mathrm{s}$. 
TABLE.II Simulation Setup Parameters

\begin{tabular}{|l|l|}
\hline Parameters & Values \\
\hline Area & $100 \times 100 \mathrm{~m}^{2}$ \\
\hline Transmission Range & $40 \mathrm{~m}$ \\
\hline $\begin{array}{l}\text { Number of randomly } \\
\text { distributed users }\end{array}$ & 10 users \\
\hline Physical/MAC layer & IEEE 802.11b at 1Mbps \\
\hline Mobility Model & $\begin{array}{l}\text { Random way point with } \\
\text { pause time }\end{array}$ \\
\hline Pause Time & $2.0 \mathrm{~s}$ \\
\hline Maximum Mobility Speed & $5,10,15,20 \mathrm{~m} / \mathrm{s}$ \\
\hline Packet size & 512 Bytes \\
\hline Traffic Type & $\begin{array}{l}\text { TCP(Transmission Control } \\
\text { Protocol) }\end{array}$ \\
\hline Simulation Time & $200 \mathrm{~s}$ \\
\hline Number of Simulation Runs & 30 \\
\hline
\end{tabular}

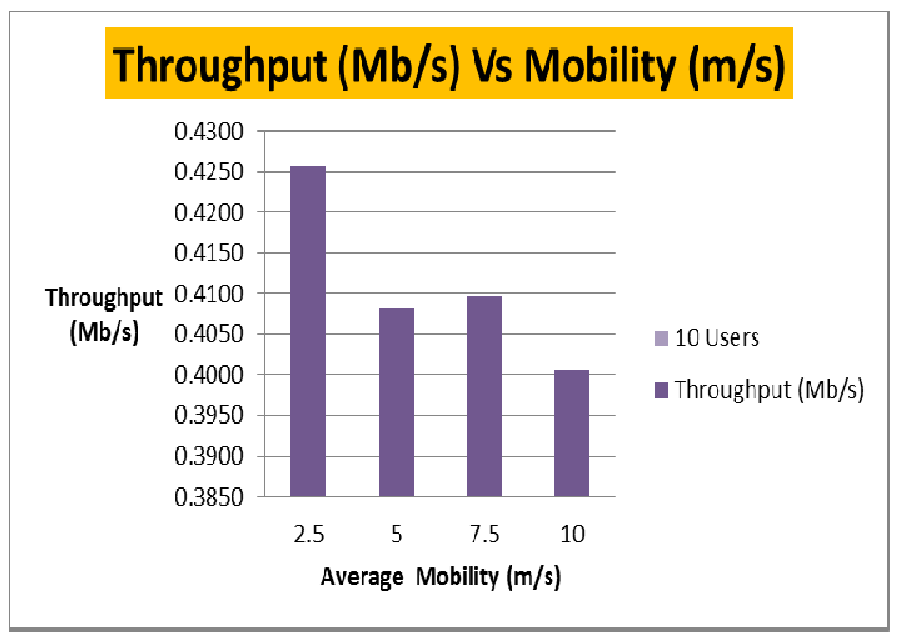

Fig.7. Average Throughput Versus Average Mobility

Fog.8.shows the effect of mobility of nodes on the PDR.We run simulation with maximum speed of $5,10,15$, and $20 \mathrm{~m} / \mathrm{s}$. For each case, we compute the average speed of nodes and run our simulation for 30 trials, each with duration time of 200s shows that at average speed of $2.5 \mathrm{~m} / \mathrm{s}$, the PDR reached its maximum value of $94.0059 \%$. A noticeable drop in the PDR of $92.7323 \%$ took place at average speed of $5 \mathrm{~m} / \mathrm{s}$. A slight increase in the PDR of $92.8258 \%$ at average speed $7.5 \mathrm{~m} / \mathrm{s}$. The least PDR took place at average speed of $10 \mathrm{~m} / \mathrm{s}$. 


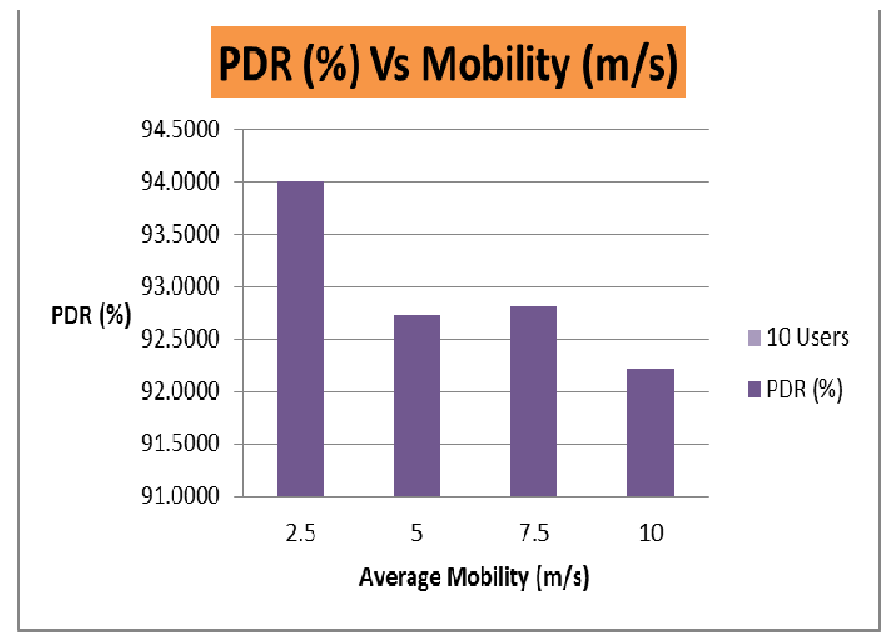

Fig.8. Average PDR versus Average Mobility

\section{CONCLUSION}

The degree of variability of the channel state of the wireless networks is quite high in comparison to wired networks. This variability is a result of several factors, one of which is the mobility of nodes. In this paper, a real time simulation was carried out with different mobility scenarios and different network loads to investigate the effect of such factors on the overall network performance. Throughput and Packet Delivery Ratio were computed to validate our simulation.

\section{REFERENCES}

[1] I.F. Akyildiz,J.S.M. Ho, and W. Wang“Mobility Management in Next-Generation Wireless Systems,’PROCEEDINGS OF THE IEEE, vol. 87, August 1999.

[2] N.P. Makwana, S.K. Vithalani, and J.D. Dhanesha, "Intrusion Detection-Watchdog: For Secure AODV Routing Protocol in VANET," International Journal of Engineering Trends and Technology, vol.4, May 2013.

[3] A. Benslimane, A. Rachedi, "Rate Adaptation scheme For IEEE 802.11-based MANETs," Journal of Network and Computer Applications, vol. 39, March 2014

[4] S.Siraj,A.K. Gupta, and R. Badgujar "Network Simulation Tools Survey,"International Journal of Advanced Research in Computer and Communication Engineering, vol. 1, June 2014.

[5] G. Anastasi, M. Conti, E. Gregori, "IEEE 802.11 AdHoc Networks, Protocols, Performance and Open Issues,".

[6] G. Miao, Y.G. Li, and A. Swami, "Channel-Aware Distributed Medium Access Control ," IEEE/ACM TRANSACTIONS ON NETWORKING,vol.20, August 2012.

[7] M. Yazid, L.B. Medjkoune, D. Ai“ssani, and L.Z. Khodja, "Analytical analysis of applying packet fragmentation mechanism on IEEE 802.11b DCF network in non ideal channel with infinite load conditions," Springer, October 2013

[8] P. Srivastava, and D. Singh, “A Survey on Modified RTS/CTS Mechanism,'International Journal of Computer Networks and Wireless Communications, vol. 3, February 2013.

[9] http://www.isi.edu/nsnam

[10] N. Sah, N.R. Prakash, and D. Bagai, "Analysis of Bandwidth Utilization for Wireless Mesh Networks,"vol.4, October 2013. 